\title{
Anomalous origin of right coronary artery from mid-left anterior descending artery leading to coronary steal phenomenon and ischemia
}

\author{
Yasar Turan ${ }^{1}$, Savaş Sarikaya ${ }^{1}$, Aylin Okur $^{2}$, Seyhan Karacavus ${ }^{3}$, Onur Akgün ${ }^{1}$ \\ ${ }^{1}$ Cardiology Department, Faculty of Medicine, Bozok University, Yozgat, Turkey \\ ${ }^{2}$ Radiology Department, Faculty of Medicine, Bozok University, Yozgat, Turkey \\ ${ }^{3}$ Nuclear Medicine Department, Faculty of Medicine, Bozok University, Yozgat, Turkey
}

Adv Interv Cardiol 2016; 12, 1 (43): 55-56 DOI: $10.5114 /$ pwki.2016.56950

Coronary anomalies are usually diagnosed incidentally during coronary angiography. Anomalous origin of the right coronary artery (RCA) from the left anterior descending (LAD) is a rare clinical entity [1, 2]. Noninvasive anatomic assessment by coronary computed tomography (CT) angiography has evolved to become a highly accurate method in the diagnosis of coronary artery disease (CAD), comparable to conventional invasive coronary angiography [3]. We report a case of anomalous RCA originating from the mid-LAD leading to exertional dyspnea.

A 50-year-old woman was admitted to our cardiology outpatient clinic with the complaint of exertional dyspnea and atypical chest pain. The patient was not eligible for the treadmill stress test due to congenital hip dislocation. Therefore, we scheduled myocardial perfusion scintigraphy, and hypoperfusion was detected in the mid and basal segments of the anterior region of the heart (Figure 1). These changes were interpreted as mild ischemia. As further investigation, coronary CT angiography was scheduled for the patient. Coronary CT angiography demonstrated a single coronary artery from the left Valsalva sinus. An anomalous RCA originated from the mid-LAD, after the first diagonal branch, coursing to the right, anterior to the pulmonary artery, then trajecting downward into the right atrioventricular groove (Figures 2-4).

Anomalies of the RCA are observed to a relatively lesser extent. Yamanaka and Hobbs [4] reported anomalous RCA at the rate of $0.26 \%$ in a series of 126,595 patients. Turkmen et al. reported the incidence of single coronary artery anomaly as $0.031 \%$ in a series of 215,140 patients who underwent routine coronary angiography [5].
Although anomalous coronary artery is considered to be a benign entity, it may cause myocardial ischemia and even sudden cardiac death. Most of these patients are asymptomatic and have normal electrocardiograms at rest. However, ischemia may be precipitated by strenuous, prolonged physical activity. Our patient's symptoms emerged only with exercise.

Myocardial ischemia should be excluded before a coronary anomaly is considered as benign. Myocardial perfusion scintigraphy is a useful and widespread method for indicating the presence of ischemia. Also, coronary CT angiography may be beneficial in the diagnosis of CAD and coronary anomalies. Coronary tomputed angi-
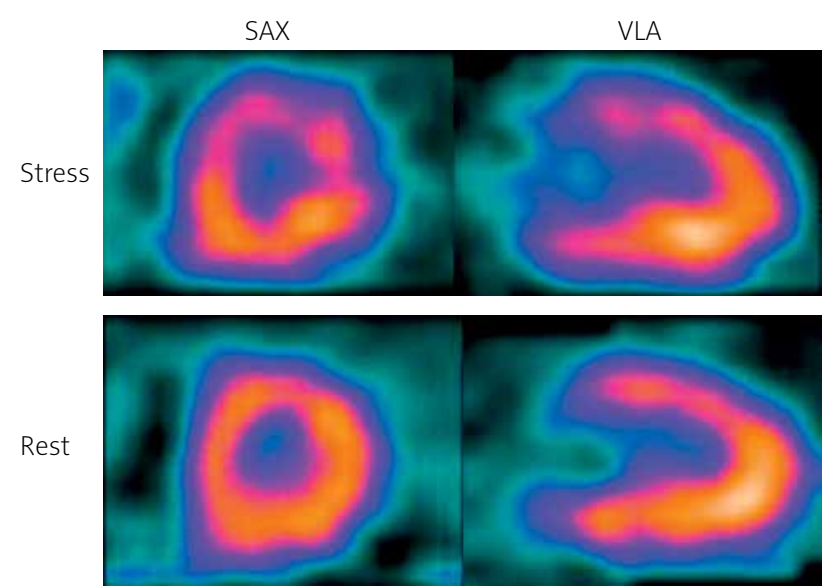

Figure 1. Tomographic scans during stress myocardial scintigraphy. Perfusion defects in the mid and basal anterior segments in the short-axis (SAX) and vertical long-axis (VLA) views. The resting SAX and VLA views show the normal perfusion

Corresponding author:

Prof. Savas Sarıkaya, Cardiology Department, Faculty of Medicine, Bozok University, Yozgat, Turkey, phone: 03542126070,

e-mail: cardiology58@gmail.com

Received: 1.07.2015, accepted: 29.07.2015. 


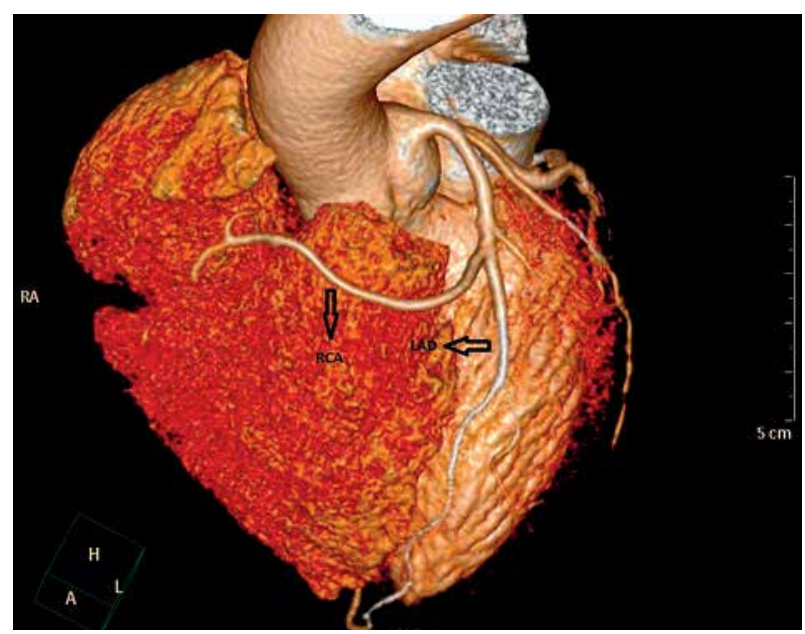

Figure 2. Coronary computed tomography angiography image showing an anomalous RCA originating from the mid-LAD and coursing anterior of the aorta and the pulmonary trunk

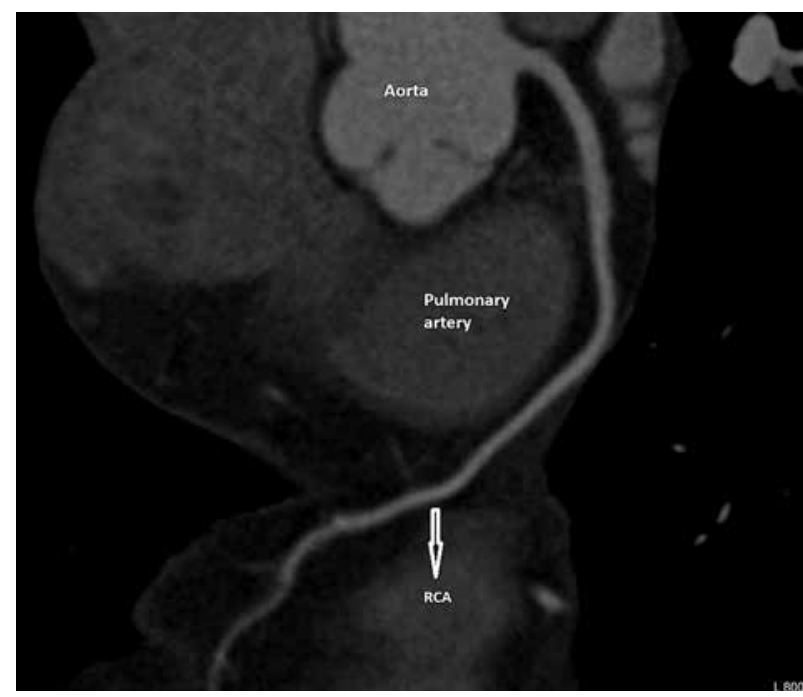

Figure 4. Anatomical course of the anomalous RCA

ography demonstrated a single coronary artery anomaly in our patient (Figures 2-4). The coronary tree was free of significant intraluminal narrowing, atherosclerosis, unusual angling or any compression sign which may explain ischemia.

In our case, exertional dyspnea may be partly secondary to the myocardial ischemia, which could result from the steal phenomenon due to single coronary artery anomaly. To the best of our knowledge, this is the first case that shows myocardial ischemia in the LAD region in single coronary artery anomaly despite absence of any significant narrowing in the coronary arteries.

\section{Conflict of interest}

The author declares no conflict of interest.

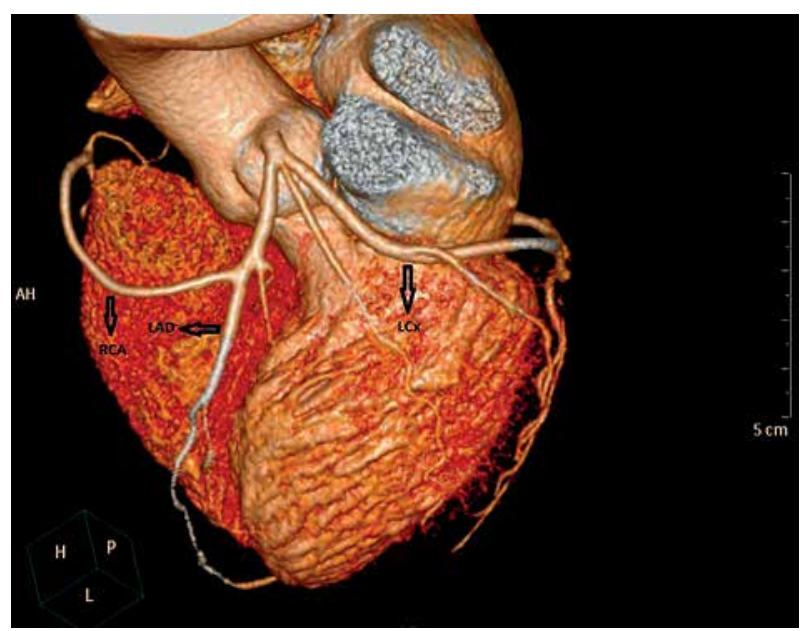

Figure 3. Overview of coronary vessel course in relation to the great arterial vessels aorta and pulmonary trunk

\section{References}

1. Teragawa H, Okada K, Sueda T. Anomalous origin of the right coronary artery from the left anterior descending coronary artery. Heart 2004; 90: 1492.

2. Jammula P, Gupta R, Uretsky BF. Anomalous origin of the right coronary artery from the left anterior descending artery. Heart 2005; 91: e30.

3. Hulten EA, Carbonaro S, Petrillo SP, et al. Prognostic value of cardiac computed tomography angiography: a systematic review and meta-analysis. J Am Coll Cardiol 2011; 57: 1237-47.

4. Yamanaka O, Hobbs RE. Coronary artery anomalies in 126,595 patients undergoing coronary arteriography. Cathet Cardiovasc Diagn 1990; 21: 28-40.

5. Turkmen S, Yolcu M, Sertcelik A, et al. Single coronary artery incidence in 215,140 patients undergoing coronary angiography. Folia Morphol (Warsz) 2014; 73: 469-74. 